\title{
COMMENTARY
}

\section{Long-term risk of sepsis among survivors of acute kidney injury}

Edward Clark ${ }^{1}$ and Sean M Bagshaw ${ }^{2^{*}}$

See related research by Lai et al., http://ccforum.com/content/17/5/R231

\begin{abstract}
Many prior studies have shown that, in critically ill patients, acute kidney injury (AKI) commonly occurs in association with sepsis and its presence portends an increased likelihood of poor outcomes. In contrast, few studies have focused specifically on the influence of AKI on the long-term risk of developing sepsis. In a previous issue of Critical Care, a population-based cohort study by Lai and colleagues reported a long-term increased risk of severe sepsis for patients surviving beyond 90 days following hospitalization with an episode of AKI requiring renal replacement therapy. While the pathophysiologic mechanisms that underpin this finding remain to be elucidated and causality cannot be proven, this study suggests that severe AKI confers long-term susceptibility to infection and focuses further attention on the critical importance of long-term surveillance for survivors of severe AKI. Further mechanistic and clinical studies are required to more precisely define the extent and duration of any increased risk of severe sepsis beyond which a need for ongoing renal replacement therapy following AKI might be associated. Nonetheless, this novel study by Lai and colleagues could lead to a number of important new avenues for clinical inquiry, such as whether it might be possible to identify those most susceptible to severe sepsis after AKI and, ultimately, whether such episodes might be preventable.
\end{abstract}

In a previous issue of Critical Care, Lai and colleagues describe a population-based cohort study evaluating the risk of severe sepsis following hospitalization associated with an episode of severe acute kidney injury (AKI) requiring renal replacement therapy (RRT) [1]. Using a large administrative database over a 10-year period,

\footnotetext{
* Correspondence: bagshaw@ualberta.ca

${ }^{2}$ Division of Critical Care Medicine (University of Alberta Hospital), Faculty of Medicine and Dentistry, University of Alberta, 2-124E Clinical Sciences Building, 8440-112 ST NW, Edmonton T6G2B7, Canada

Full list of author information is available at the end of the article
}

patients with severe AKI receiving RRT who survived to at least 90 days after hospital discharge were matched to those without severe AKI and followed for approximately 4 years for the occurrence of severe sepsis. The adjusted risk of developing severe sepsis prompting hospitalization was approximately twofold higher among those having had severe AKI compared with those not having had AKI (incidence rate of 6.84 vs. 2.32 per 100 person-years). Importantly, even among survivors with severe AKI who recovered kidney function to become dialysis independent, the adjusted risk of severe sepsis remained 50 to $70 \%$ higher compared with matched patients with no history of AKI.

AKI remains a widespread complication of acute illness, occurring in one in five adults and one in three children in association with an acute hospitalization [2]. Trends from observational data provide compelling evidence that the incidence of AKI continues to grow. While mortality has concomitantly decreased, more patients are ultimately suffering the long-term sequelae of AKI [3]. These sequelae include increased long-term risks of death, chronic kidney disease (CKD), progression to end-stage kidney disease, reduced quality of life and excess resource utilization $[4,5]$.

Prior data have shown that AKI in critical illness most commonly occurs in association with sepsis and that its presence clearly worsens the risk of adverse outcomes [6]. However, fewer studies have focused specifically on any increased risks imposed by AKI for the downstream development of sepsis and consequent morbidity and mortality [7-11]. One of the earliest studies to show an increased risk of sepsis after AKI was performed in a cohort of hospitalized patients suffering AKI following radiocontrast procedures [11]. Among nonsurvivors, $45 \%$ developed a new episode of sepsis. The risk of nosocomial infection is higher among patients developing AKI after cardiac surgery [10]. Similarly, nosocomial infections occur more commonly among critically ill patients with severe AKI requiring RRT compared with 
matched controls $[7,9]$. A recent cohort study noted that sepsis after any AKI occurred in $40 \%$ of critically ill patients after a median 5 days [8]. Among critically ill patients with severe AKI receiving RRT, 13.4\% of nosocomial infections occurred after discontinuation of RRT [9]. Sepsis after AKI portends a higher risk of RRT utilization and death and higher resource utilization $[8,10]$. Indeed, among nonsurvivors hospitalized with AKI, sepsis was the most common attributable cause of death, occurring in $41 \%$ [12].

These data clearly establish AKI as an immediate and short-term risk factor for the development of nosocomial infections and sepsis. This specific short-term risk among critically ill patients would appear understandable; however, the precise pathophysiologic mechanisms whereby AKI increases susceptibility for sepsis remains incompletely understood. There is probably a complex interplay of modifying factors including premorbid conditions that are also associated with higher risk of infection, such as genetics (for example, gene polymorphisms in inflammatory mediators), older age and comorbidities (for example, CKD, cancers), as well as more proximate factors including acute immune dysregulation, acute organ dysfunction, and treatment-related risks (for example, mechanical ventilation, hemodialysis catheter).

The data described by Lai and colleagues do not inform about the short-term risk of severe sepsis after AKI [1]. Instead, their study specifically evaluated the longerterm risk of severe sepsis conditional on survival to 90 days after index hospitalization. While nonmodifiable risk factors such as genetics, age, comorbidity and ongoing treatment-related risks are probably still contributing factors, the biologic mechanisms whereby AKI confers longer-term susceptibility to infection and sepsis are unknown. Nonetheless, the AKI and infection susceptibility association is plausible given that acute kidney insults are known to induce changes in gene regulation, oxidative stress, inflammation, leukocyte trafficking, and apoptosis and to incite systemic and distal organ injury [13].

While the idea that AKI independently confers increased risk of severe sepsis long after the occurrence of AKI is conceptually appealing, there are some caveats to consider. The hypothesis posited by Lai and colleagues is that AKI confers a sustained susceptibility to the development of new infections or severe sepsis through persistent immune suppression or senescence. While the study did show an increased risk of severe sepsis for survivors who recovered from AKI, the risk of severe sepsis among RRT-dependent survivors may largely be explained by RRT dependence itself. Sepsis remains a leading cause of morbidity and mortality among end-stage kidney disease patients: the rate of sepsis for US chronic hemodialysis patients was recently reported to be
282 per 1,000 patient-years [14]. Similarly, persistent nondialysis-requiring CKD could serve to confound any association between AKI and increased susceptibility to sepsis for those who recovered to become dialysis independent. Maladaptive repair mechanisms after AKI and incident CKD have been shown to modify the long-term risk of developing severe sepsis $[15,16]$.

The study by Lai and colleagues has appropriately focused attention on the critical importance of long-term surveillance of survivors of AKI and may ultimately generate a number of important additional avenues of inquiry. If the risk of downstream severe sepsis is increased among survivors of severe AKI, can we identify those most susceptible and are such episodes amenable to preventative interventions? First, additional mechanistic and clinical studies are needed to better define the nature, magnitude and duration of the risk of severe sepsis following AKI, independent of the risks conferred by either CKD or end-stage kidney disease that requires ongoing RRT.

\section{Abbreviations \\ AKl: Acute kidney injury; CKD: Chronic kidney disease; RRT: Renal replacement therapy.}

\section{Competing interests}

The authors declare that they have no competing interests.

\section{Acknowledgements}

SMB is supported by a Canada Research Chair in Critical Care Nephrology and Clinical Investigator Award from Alberta Innovates - Health Solutions.

\section{Author details}

${ }^{1}$ Kidney Research Centre - Ottawa Hospital Research Institute, University of Ottawa, 725 Parkdale Avenue, Ottawa, Ontario K1Y 4E9, Canada. ${ }^{2}$ Division of Critical Care Medicine (University of Alberta Hospital), Faculty of Medicine and Dentistry, University of Alberta, 2-124E Clinical Sciences Building, 8440-112 ST NW, Edmonton T6G2B7, Canada.

\section{Published: 24 Jan 2014}

\section{References}

1. Lai TS, Wang CY, Pan SC, Huang TM, Lin MC, Lai CF, Wu CH, Wu VC, Chien KL, National Taiwan University Hospital Study Group on Acute Renal Failure (NSARF): Risk of developing severe sepsis after acute kidney injury: a population-based cohort study. Crit Care 2013, 17:R231.

2. Susantitaphong P, Cruz DN, Cerda J, Abulfaraj M, Alqahtani F, Koulouridis I, Jaber BL, Acute Kidney Injury Advisory Group of the American Society of Nephrology: World incidence of AKI: a meta-analysis. Clin J Am Soc Nephrol 2013, 8:1482-1493.

3. Xue JL, Daniels F, Star RA, Kimmel PL, Eggers PW, Molitoris BA, Himmelfarb J, Collins AJ: Incidence and mortality of acute renal failure in Medicare beneficiaries, 1992 to 2001. J Am Soc Nephrol 2006, 17:1135-1142.

4. Joyce VR, Smith MW, Johansen $\mathrm{KL}$, Unruh ML, Siroka AM, O'Connor TZ, Palevsky PM, Veteran Affairs/National Institutes of Health Acute Renal Failure Trial Network: Health-related quality of life as a predictor of mortality among survivors of AKI. Clin J Am Soc Nephrol 2012, 7:1063-1070.

5. Lameire NH, Bagga A, Cruz D, De Maeseneer J, Endre Z, Kellum JA, Liu KD, Mehta RL, Pannu N, Van Biesen W, Vanholder R: Acute kidney injury: an increasing global concern. Lancet 2013, 382:170-179.

6. Bagshaw SM, George C, Bellomo R: Early acute kidney injury and sepsis: a multicentre evaluation. Crit Care 2008, 12:R47.

7. Hoste EA, Blot SI, Lameire NH, Vanholder RC, De Bacquer D, Colardyn FA: Effect of nosocomial bloodstream infection on the outcome of critically 
ill patients with acute renal failure treated with renal replacement therapy. J Am Soc Nephrol 2004, 15:454-462.

8. Mehta RL, Bouchard J, Soroko SB, Ikizler TA, Paganini EP, Chertow GM, Himmelfarb J, Program to Improve Care in Acute Renal Disease (PICARD) Study Group: Sepsis as a cause and consequence of acute kidney injury: Program to Improve Care in Acute Renal Disease. Intensive Care Med 2011, 37:241-248

9. Reynvoet E, Vandijck DM, Blot SI, Dhondt AW, De Waele JJ, Claus S, Buyle FM, Vanholder RC, Hoste EA: Epidemiology of infection in critically ill patients with acute renal failure. Crit Care Med 2009, 37:2203-2209.

10. Thakar CV, Yared JP, Worley S, Cotman K, Paganini EP: Renal dysfunction and serious infections after open-heart surgery. Kidney Int 2003, 64:239-246.

11. Levy EM, Viscoli CM, Horwitz RI: The effect of acute renal failure on mortality. A cohort analysis. JAMA 1996, 275:1489-1494.

12. Selby NM, Kolhe NV, Mclntyre CW, Monaghan J, Lawson N, Elliott D, Packington R, Fluck RJ: Defining the cause of death in hospitalised patients with acute kidney injury. PLoS One 2012, 7:e48580.

13. Grams ME, Rabb H: The distant organ effects of acute kidney injury. Kidney Int 2012, 81:942-948.

14. 2013 USRDS annual data report: atlas of end-stage renal disease in the United States. [http://www.usrds.org/2013/slides/indiv/v2index.html]

15. Doi K, Leelahavanichkul A, Hu X, Sidransky KL, Zhou H, Qin Y, Eisner C, Schnermann J, Yuen PS, Star RA: Pre-existing renal disease promotes sepsis-induced acute kidney injury and worsens outcome. Kidney Int 2008, 74:1017-1025.

16. Zager RA: 'Biologic memory' in response to acute kidney injury: cytoresistance, toll-like receptor hyper-responsiveness and the onset of progressive renal disease. Nephrol Dial Transplant 2013, 28:1985-1993.

$10.1186 / \operatorname{cc} 13708$

Cite this article as: Clark and Bagshaw: Long-term risk of sepsis among survivors of acute kidney injury. Critical Care 2014, 18:103 